\title{
Naphazoline Hydrochloride
}

National Cancer Institute

\section{Source}

National Cancer Institute. Naphazoline Hydrochloride. NCI Thesaurus. Code C47632.

The hydrochloride salt form of naphazoline, an imidazole derivative and a direct-acting sympathomimetic amine with vasoconstrictive properties. Upon ocular administration, naphazoline hydrochloride exerts its effect by acting on alpha-adrenergic receptors in the arterioles of the conjunctiva to produce vasoconstriction, resulting in decreased conjunctival congestion and diminished itching, irritation and redness. 